\title{
Measuring the Mechanical Properties of Laminated Wood Structures Using a Homemade Bending Tester
}

\author{
J. Weber, and A. J. Stollenwerk* \\ University of Northern Iowa, Department of Physics, 215 Begeman Hall, Cedar Falls, Iowa 50614-0150 \\ Students: weberjaw@uni.edu \\ Mentor: andrew.stollenwerk@uni.edu
}

\begin{abstract}
The choice of materials used to build a laminate recurve bow is crucial to optimizing performance. To this end, a low-cost bending tester was designed and built to measure the flexural modulus and modulus of rupture on a variety of wooden laminates. To gain a better understanding of the relationship between the properties of the laminates and the individual species comprising the laminates, woods with a wide range of elastic and strength properties were chosen. Differences between the expected and experimental results are attributed to the properties of the adhesive and defects in the wood.
\end{abstract}

\section{KEYWORDS}

bending test; recurve bow; wood; laminates; modulus of rupture; flexural modulus

\section{INTRODUCTION}

Spanning most cultures around the world, numerous types of bow have been developed to make use of available resources or meet specific needs. Each design has in common a pair of elastic limbs connected by a string attached at the nocking points located at either end of the bow. The side of the limb facing the string is known as the belly of the bow, the other side is known as the backing. Pulling the string back deforms the limbs, creating a compression in the belly and tension in the backing. Potential energy builds up in the limbs with increasing deformation and is converted to kinetic energy when released. Perhaps the simplest way to build a bow is to shape one from a single piece of wood, known as a self bow. Self bows are usually straight when unstrung such as the English longbow. In contrast to a straight bow, a recurve bow has limbs that curve away from the archer when unstrung. A comparison between a straight self bow and a recurve bow in unstrung, strung, and fully drawn states is shown in Fig. 1. The geometry of the recurve causes the lever arm of the limb to increase as it is flexed so that the force necessary to draw the bow increases rapidly at first before slowing quickly. Due to this change in mechanical advantage, a recurve is able to more efficiently store energy than a straight bow for the same force used to fully draw the bow. However, this places greater strain on the limbs, making the choice of materials crucial to success.

Modern recurve limbs are usually laminates created by bonding thin layers of material together with an adhesive. This process allows one to create the desired shape of the recurved limbs by clamping the layers in a mold as the adhesive dries. The materials comprising the laminate are chosen to optimize certain desired qualities. For example, materials capable of withstanding large amounts of stress due tension are ideal for the backing of the bow and those with good compression properties are desirable for the belly. A poor choice of materials can lead to mechanical failure via yield or fracture. A material yields when it undergoes a non-linear deformation and is typically caused by structural changes such as buckling of compressive fibers. However, bows typically fail due to a fracture characterized by a catastrophic splitting of the material due to tensile stress. The maximum tensile stress in a material before failure can be quantified by the modulus of rupture. Due to the way a bow propels an arrow, the elastic properties of the limbs are also of interest. The amount of force necessary to draw a bow with given dimensions can be adjusted by 


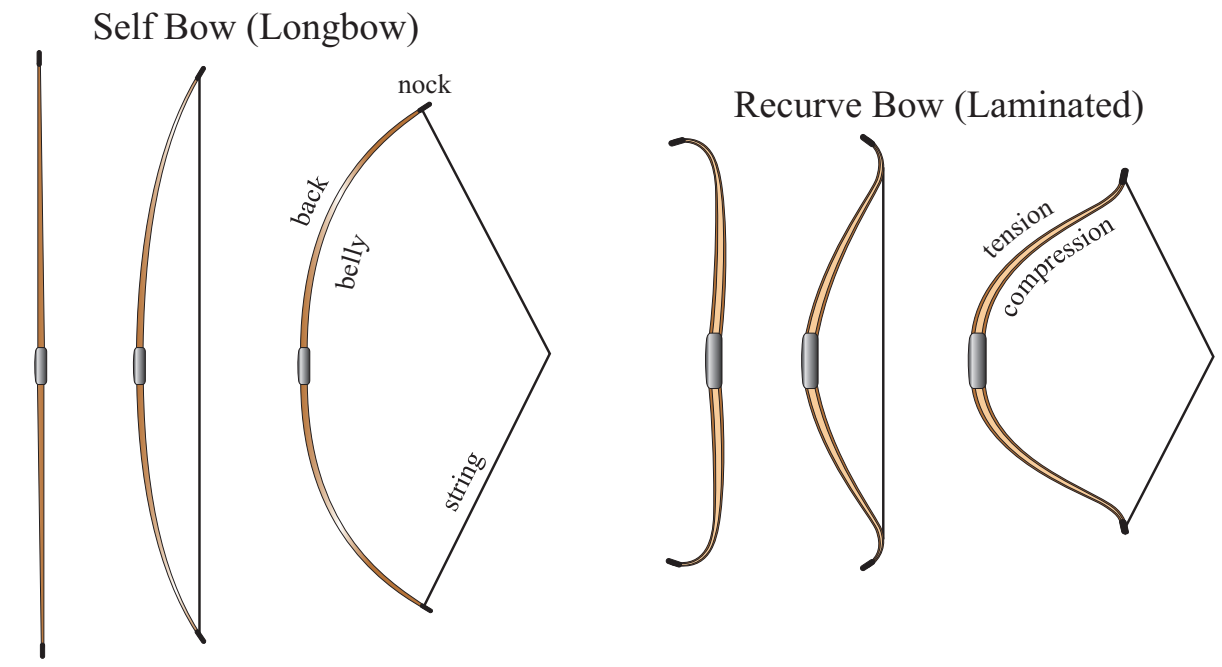

Figure 1. A comparison of a straight self bow and a recurve bow in unstrung, strung, and fully drawn states.

using materials with different flexural moduli, or resistance to bending. Most recurve bows are made by laminating a wood or carbon core with fiberglass to achieve these desired qualities. ${ }^{\mathbf{1}, \mathbf{2}}$ It is possible to build a recurve bow using only wooden layers, but care must be made when choosing the layers given the variability of properties among different species of wood.

In the this paper, a low-cost bending tester was designed and built to measure the flexural modulus and the modulus of rupture of wooden laminates constructed from several different species of woods. The flexural modulus was compared to expected values calculated from the properties of the individual species comprising the laminate. The modulus of rupture was compared to that of the species used as the backing layer.

\section{METHODS AND PROCEDURES}

A number of different wood species were purchased from a mill to fabricate the laminates: Peltogyne spp. (purple heart), Anadenanthera colubrina (curupay), Hymenaea courbaril (jatoba), Handroanthus spp. (ipe), Acer rubrum (red maple), and Carya laciniosa (shellbark hickory). Curupay, ipe, and hickory were selected as backing material due to their reported high moduli of rupture ${ }^{3}$ and availability. Other woods were selected to include a wide variety of elastic properties ${ }^{3}$ to explore how the flexural moduli of individual woods affect the overall stiffness of the combined layers. A table saw was used to cut kiln dried lumber into strips with cross-sectional dimensions of approximately $5 \times$ $35 \mathrm{~mm}$ and varying lengths. Given the effect on mechanical properties ${ }^{4}$, effort was made to ensure that the overall grain was parallel to the length of the strips to within $5^{\circ}$ in either direction. A thickness planer was used to smooth both sides and create a uniform thickness of $3.3 \mathrm{~mm}$ and width of $34 \mathrm{~mm}$. A number of laminates were created using various combinations of wood species. Each laminate stack consisted of three strips of wood bonded together with Titebond III ${ }^{\circledR}$ wood glue applied after lightly sanding the surface with 120 grit sand paper and removing any dust with a damp cloth. When used as a backing layer, Ipe stripes were cleaned using acetone to remove natural oils and increase adhesion. ${ }^{3}$ Laminates were compressed using spring clamps spaced approximately $2 \mathrm{~cm}$ as the glue cured over a 24 hour period. The final thickness after a 24 hour curing period was approximately $10.0 \pm 0.2 \mathrm{~mm}$. Laminated stacks were cut into sizes with width of approximately $15 \mathrm{~mm}$ and length of $16 \mathrm{~cm}$.

The mechanical properties of each sample were measured using a homemade three point bending tester. This setup is depicted in Fig. 2 and was used as a low-cost alternative to more expensive, commercially available systems not widely available at undergraduate institutions. A web strap, hand-operated winch was used to apply a force to the middle of the board via a flat U-bolt in series with an American Weigh TL-330 hanging scale with an accuracy of 


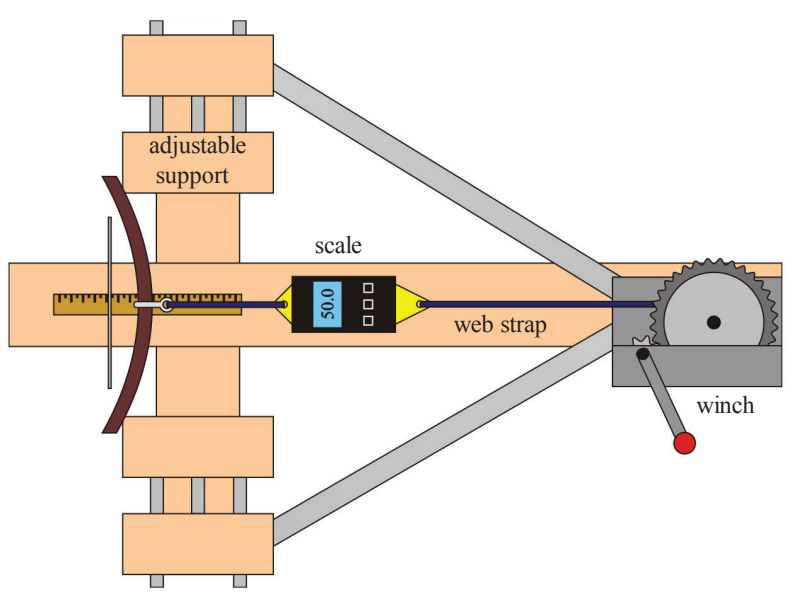

Figure 2. Schematic of the homemade three point bending setup.

$\pm 0.3 \mathrm{~kg}$. Samples of different lengths can be accommodated with adjustable support structures. Depending on the amount of stretch in the web strap, the sample was pulled back in increments of roughly $1.0-2.0 \mathrm{~mm}$ as measured using electronic calipers. The maximum displacement of the laminates prior to failure was also measured using electronic calipers. The maximum force up to sample failure was logged on the electronic scale.

The displacement and maximum force were used to calculate the flexural modulus and the modulus of rupture for each sample. For a sample with a rectangular cross section, the flexural modulus can be calculated from the displacement due to a force applied to the middle of the sample lengthwise, ${ }^{\mathbf{5}}$

$$
E_{i}=\frac{L^{3} F}{4 w t^{3} d}
$$

Here $F$ is the applied force, $d$ is the displacement, $L$ is the length of the sample, $w$ is the width, and $t$ is the thickness. The modulus of rupture for the same sample is determined from the maximum force applied prior to sample failure, ${ }^{6}$

$$
\sigma=\frac{3 F L}{2 w t^{2}}
$$

The effective flexural modulus for a multi layer laminate can be calculated from the flexural moduli of the comprising layers $\left(E_{i}\right)$ as, ${ }^{7}$

$$
E=\frac{\sum_{n=1}^{3} E_{i} I_{i}}{I_{t}}
$$

The area moment of inertia of each layer $\left(I_{i}\right)$ and the total area moment of inertial $\left(I_{t}\right)$ are determined relative to the neutral axis defined as the axis where the force acting on the cross-section is zero when the material is bent, ${ }^{\mathbf{8}}$

$$
\bar{y}=\frac{\sum_{n=1}^{3} y_{i} A_{i}}{A_{t}}
$$

Here $y_{i}$ is the distance from the centroid of each layer to the base of the laminate. The cross sectional area of each layer $\left(A_{i}\right)$ and the total cross sectional area $\left(A_{t}\right)$ are calculated using the principle of equivalent width where each layer is assumed to be of the same material with a corresponding width adjusted such that this homogeneous laminate has the same mechanical properties as the original laminate. For example, if the flexural modulus of material A is twice that of a material B then the equivalent of material A would be that of material B with half the width. The 
area moment of inertia of each rectangular layer ${ }^{8}$ is calculated using the parallel axis theorem ${ }^{9}$ from the height $(h)$ and the equivalent width $(w)$,

$$
I_{i}=\frac{1}{12} w h^{3}+A_{i}\left(y_{i}-\bar{y}\right)^{2}
$$

\section{RESULTS AND DISCUSSION}

To have a basis for comparison, the mechanical properties were measured on individual species using a single strip of wood with a cross section of $3.3 \times 34 \mathrm{~mm}$ and length of $16 \mathrm{~cm}$. The results are summarized in Table 1 and are generally smaller than those found in literature by about $20 \%^{3}$ with the exception of curupay which is approximately $60 \%$ smaller than previous measurements. ${ }^{3}, 10$ This is not surprising given that each piece of wood is unique to the growth environment of the tree. ${ }^{11}$ Although attempts were made to ensure a parallel orientation, waviness in the grain around imperfections could create localized weak points. ${ }^{4}, 11$ The grain of the curupay used in this study was especially wavy compared to the other woods and is likely the reason for the large discrepancies as compared to values found in literature. Other factors may include differences in moisture content. ${ }^{12}$ While moisture content was not measured here, all samples were stored and tested in a controlled environment.

\begin{tabular}{|l||c|c|}
\hline Species & Flexural modulus $(\mathrm{GPa})$ & Modulus of rupture $(\mathrm{MPa})$ \\
\hline Purple heart & $13.1 \pm 0.6$ & $116.6 \pm 4.8$ \\
Curupay & $9.3 \pm 0.6$ & $95.7 \pm 5.0$ \\
Jatoba & $2.6 \pm 0.1$ & $70.1 \pm 2.8$ \\
Ipe & $15.0 \pm 0.7$ & $129.1 \pm 5.1$ \\
Red maple & $4.3 \pm 0.3$ & $68.0 \pm 3.2$ \\
Shellbark hickory & $5.6 \pm 0.4$ & $91.3 \pm 3.8$ \\
\hline
\end{tabular}

Table 1. Mechanical properties of individual species cut from a single piece of wood.

The flexural moduli for a variety of three layer laminates are plotted in Fig. 3 as filled squares. The open circles are the corresponding effective flexural moduli calculated from Eq. 3 using the dimensions of the woods comprising the laminate stack and the flexural moduli listed in Table 1. As seen in Fig. 3, the calculated effective flexural moduli consistently overestimate the experimental results to varying degrees. The reason for this difference may due to the glue, which is not included when calculating the effective flexural moduli. According to Eq. 3, the relatively thin layers of the glue are not expected to greatly contribute to the effective flexural modulus. However, the elasticity of the glue could permit a lateral shift between the layers of wood when bent. The greater the shift, the more the laminate will behave as three detached layers, resulting in a lower overall flexural modulus. In order to examine the effect of the glue on these samples, the flexural moduli was measured on triple layer laminates made from a single species. The resulting flexural moduli of homogenous laminates made from hickory, curupay, and ipe are 5.0 \pm 0.4 , $5.8 \pm 0.4$, and $11.3 \pm 1.7 \mathrm{GPa}$, respectively. In each case, the results are lower than those presented in Table 1, confirming that the glue itself plays a role in shaping the elastic properties of the samples.

The moduli of rupture of the laminate structures are plotted in Fig. 4 (filled squares). All samples in the present study fractured due to tensile stress. Because tensile stress is greatest along the outer curve, these data are compared to the moduli of rupture associated with the species used for the backing (open circles). In theory, these values should be equal since a tensile fracture is dependent only on the strength of the outer most fibers. The hickory and ipe backed samples are largely consistent with the single species hickory results. The curupay backed samples are somewhat less than their single species counterparts. Although the surface was lightly sanded as recommended, the high density of curupay presents known challenges when gluing. ${ }^{3}$ If a section of wood were to delaminate under strain, the sudden increase in tensile stress in this region would hasten failure. Visual inspection shows no obvious signs of delamination, but does not necessarily rule out poor bonding on a local scale that may weaken a small portion of the sample. For the most part, failures appear to be the result of a fracture of the outer fibers of the backing layer or a defect in the 


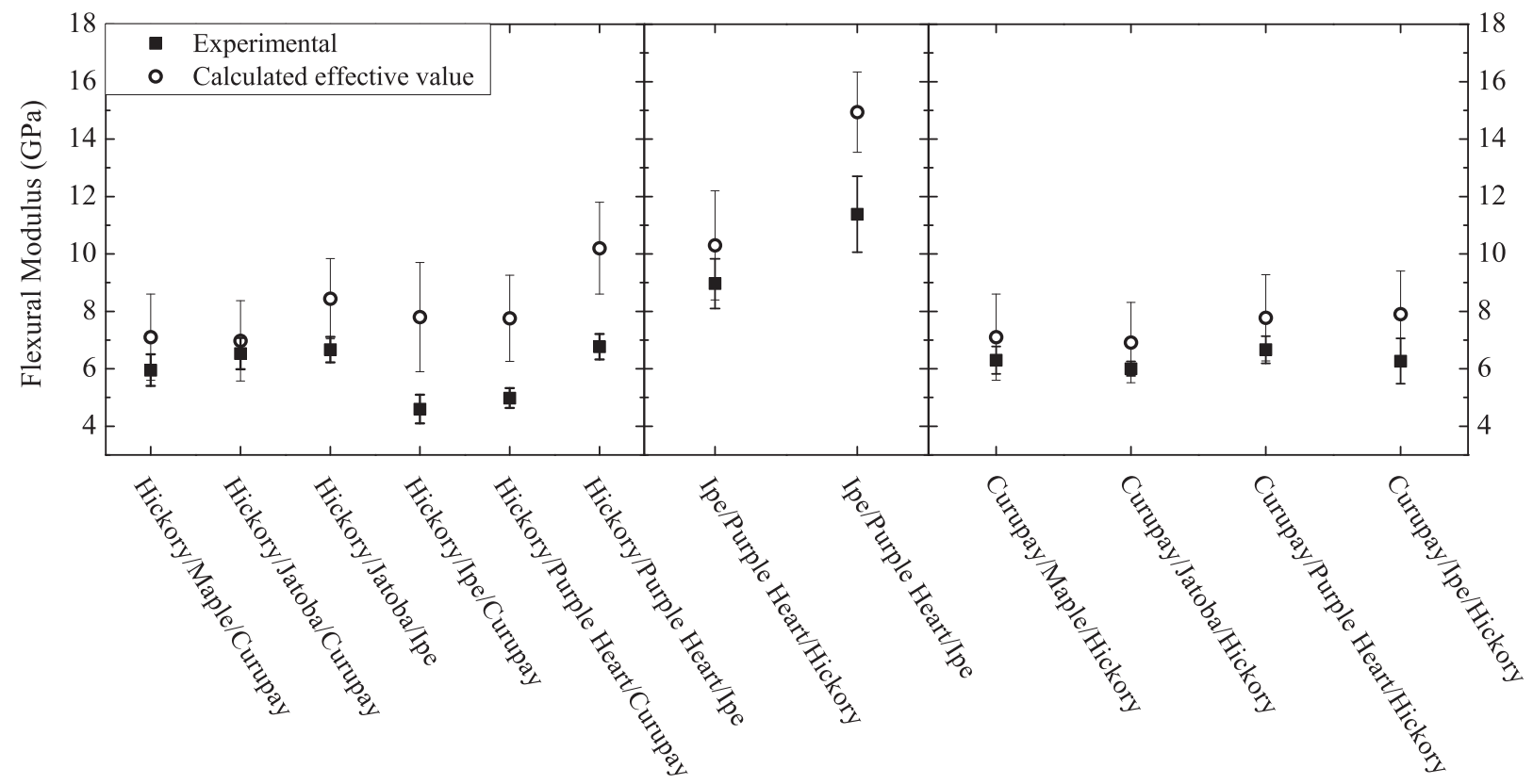

Figure 3. The effective flexural modulus of several non-homogeneous, triple layer laminates (closed squares) as compared to the effective flexural moduli (open circles) calculated from Eq. 3 using the values in Table 1.

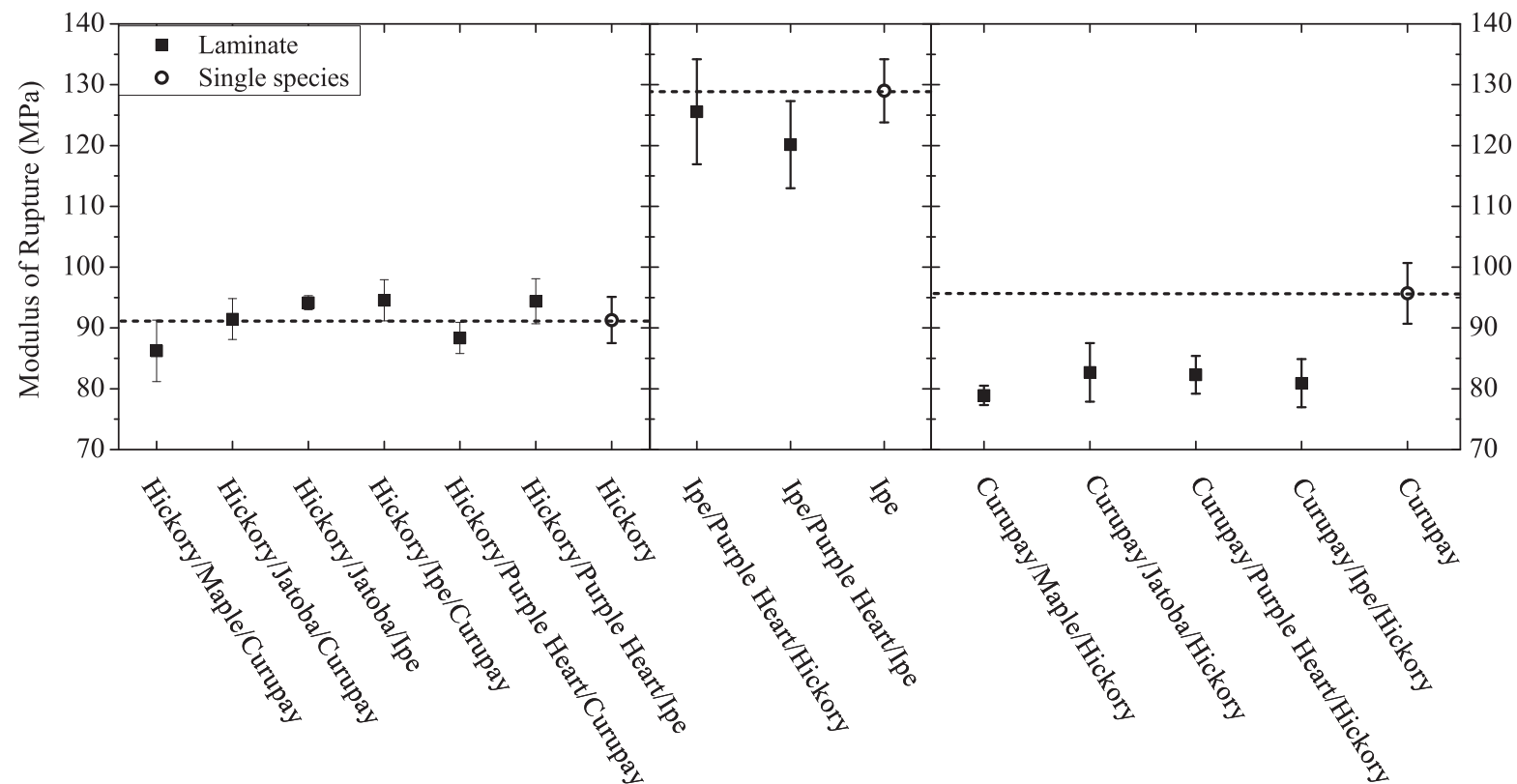

Figure 4. Modulus of rupture of non-homogeneous triple layer laminates compared to the value associated with species used for the backing layer.

grain structure that results in a crack that propagates through one of more layers. It would be interesting to repeat these experiments using curupay woods with different grain quality.

\section{CONCLUSIONS}

When choosing a wood to serve as the backing of a laminate bow it is important to have the highest possible modulus of rupture to create a strong limb. However, a strong backing won't be effective without a strong bond between layers, minimal defects in the wood, and a careful alignment of the grain. Aside from the backing, other layers can 
be chosen to tailor the properties of the bow for specific requirements. For example, a shorter bow must have thinner limbs to allow for the increased curvature when the bow is drawn. To maintain a large draw force in the thinner limbs additional layers should have a large flexural modulus.

\section{ACKNOWLEDGEMENTS}

This work was funded by a Student Opportunity for Academic Research (SOAR) grant through the University of Northern Iowa. The authors also thank C. K. Stollenwerk and M. D. Timmer for technical support.

\section{REFERENCES}

1. W. Marlow, American Journal of Physics 49, 320 (1981).

2. D. L. T. Schreiber et al., 4-H Archery Member Manual (2009).

3. E. Meier, Wood!: Identifying and Using Hundreds of Woods Worldwide (Wood Database, 2016).

4. R. B. Hoadley, Understanding wood: a craftsman's guide to wood technology (Taunton press, 2000).

5. C. Zweben, W. Smith, and M. Wardle, in Composite Materials: Testing and Design (Fifth Conference) (ASTM International, 1979).

6. R. Reeve, Methods of Soil Analysis. Part 1. Physical and Mineralogical Properties, Including Statistics of Measurement and Sampling, 466 (1965).

7. S. Girardon, L. Denaud, G. Pot, and I. Rahayu, Annals of Forest Science 73, 615 (2016).

8. R. M. Jones, Mechanics of composite materials (CRC press, 2014).

9. A. Abdulghany, American Journal of Physics 85, 791 (2017).

10. M. Chudnoff et al., Tropical timbers of the world, 607 (US Department of Agriculture, Forest Service Washington, DC, 1984).

11. D. Green, J. Winandy, and D. Kretschmann, Wood handbook: wood as an engineering material. USDA Forest Service, Forest Products Laboratory, Madison, WI, General technical report , 4 (1999).

12. C. C. Gerhards, Wood and Fiber Science 14, 4 (1982).

\section{ABOUT THE STUDENT AUTHOR}

Jacob Weber recently graduated from the University of Northern Iowa with a B.A. in Physics and is currently teaching at Bondurant-Farrar high school in Bondurant, IA

\section{PRESS SUMMARY}

This manuscript compares the mechanical properties of several different non-homogeneous laminates fabricated from an assortment of wood species. To varying degrees, the elastic properties of the laminates were less than expected due to the elastic properties of the adhesive. The strengths were largely consistent with the properties of the outermost laminate species. 